\title{
Intestinal obstruction caused by splenosis at the rectosigmoid junction, mimicking malignant pelvic tumor
}

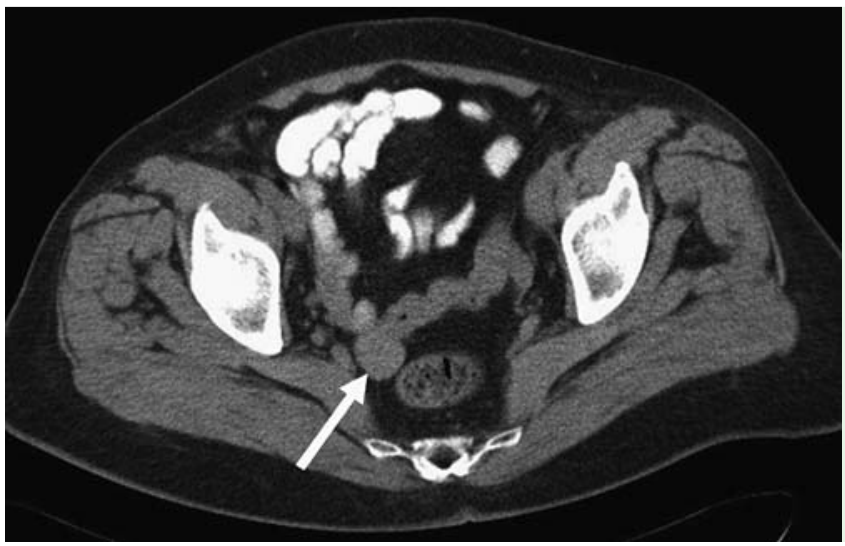

Fig. 1 Contrast-enhanced computed tomography (CT) scan showing a smoothedged, clearly demarcated, solid, expansive process with marked contrast enhancement.

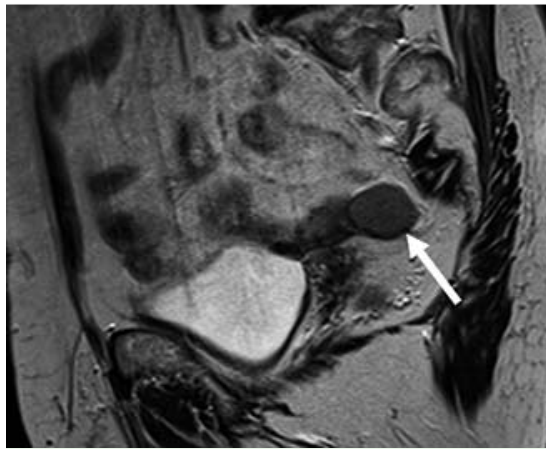

Fig. 2 T2-weighted magnetic resonance imaging (MRI) showing a solid, clearly demarcated, smooth-edged expansive process.

A 74-year-old woman presented with an incidental finding on computed tomography (CT) scanning of a tumor in the lesser pelvis of unclear origin. Questioning of the patient revealed a history of longterm, intermittent constipation and splenectomy 18 years previously after a car accident. A contrast-enhanced CT scan showed a solid expansive process with marked contrast enhancement ( $\bullet$ Fig. $\mathbf{1}$ ). Magnetic resonance imaging (MRI) revealed a $2.5-\mathrm{cm}$ clearly demarcated nodular lesion at the rectal fold, directly adjacent to the rectum ( $\bullet$ Fig. 2 ). Our suspicion was that this lesion was a neoplasm.

Colonoscopy showed a stenosis of the lumen due to extramural compression $(\bullet$ Fig. 3). This luminal compression, together with the unknown origin of the expansive process, the symptoms of intermittent constipation, and the risk of possi-

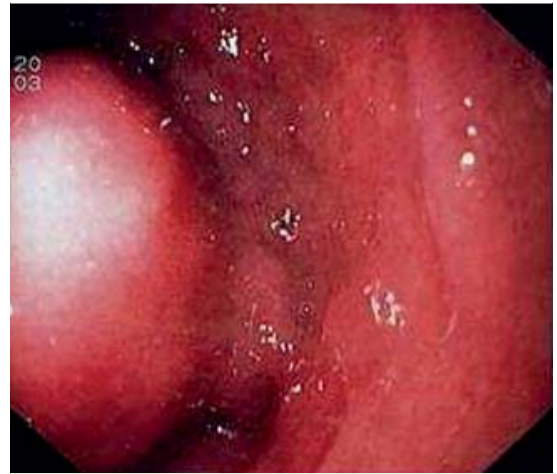

Fig. 3 Colonoscopic view showing stenosis of the lumen at the rectosigmoid junction.

ble bowel obstruction were clear indications for surgery.

A circumscribed tumor in tight contact with the proximal third of the lumen was removed without opening the lumen. The final histological examination revealed splenic tissue of typical appearance, consistent with a diagnosis of splenosis. The patient recovered uneventfully and was discharged after 5 days.

Splenosis is defined as autotransplantation of ectopic splenic tissue following splenic trauma, iatrogenic injury, or splenectomy [1]. It is an uncommon diagnosis and very often an incidental finding. If correctly diagnosed and asymptomatic, it requires no further treatment or surgery. The challenge lies however in reaching the correct diagnosis, which is often complicated by the similarity of the splenosis to a malignant process. This difficulty is confirmed by the large number of reports in which splenosis has been confused with a variety of other processes including abdominal lymphoma [2], hepatocellular carcinoma [3], and even a thoracic schwannoma [4].

The problem of differentiating between benign and malignant lesions increases even more the importance of the preoperative investigations and a detailed patient history. Although splenosis usually does not require surgical exploration, the dimensions of a lesion or its positioning within a critical area, as in our case, can carry a high risk of potential complications.

Endoscopy_UCTN_Code_CCL_1AD_2AC

Competing interests: None

V. Gincu ${ }^{1}$, P. Kornprat ${ }^{1}$, F. Thimary ${ }^{2}$, S. Jahn ${ }^{3}$, H. J.Mischinger ${ }^{1}$

Division of General Surgery, Department of Surgery, Medical University of Graz, Austria

2 Division of General Radiological Diagnostics, Department of Radiology, Medical University of Graz, Austria

3 Department of Pathology, Medical University of Graz, Austria

\section{References}

1 Buchbinder JH, Lipkoff CJ. Splenosis: multiple peritoneal splenic implants following abdominal injury. Surgery 1939; 6: 927-930

2 Priola AM, Picciotto G, Priola SM. Diffuse abdominal splenosis: a condition mimicking abdominal lymphoma. Int J Hematol 2009; 90: $543-544$

3 Kim KA, Park CM, Kim CH et al. An interesting hepatic mass: splenosis mimicking a hepatocellular carcinoma. Eur Radiol 2003; 13: $2713-2715$

4 Alaraj AM, Chamoun RB, Dahdaleh NS et al. Thoracic splenosis mimicking thoracic schwannoma: case report and review of the literature. Surg Neurol 2005; 64: 185 - 188; discussion 188

Bibliography

DOI $10.1055 / \mathrm{s}-0030-1256522$

Endoscopy 2011; 43: E260

(c) Georg Thieme Verlag KG Stuttgart · New York . ISSN 0013-726X

\section{Corresponding author}

\section{Gincu, MD}

Division of General Surgery,

Department of Surgery, Medical University of Graz Auenbruggerplatz 29

A-8036 Graz

Austria

Fax: +43-316-38514666

vasile.gincu@klinikum-graz.at 